it usually takes much less than 10 galactic revolutions (less than 10 per cent of the age of the Universe) for a pair of disks to merge to a single static entity following their initial close approach. Thus, there has been sufficient time for most galaxies to have undergone a merger during the history of the Universe and yet for less than 10 per cent of galaxies at any one time to show obvious signs (such as two nuclei) of recent violent interactions. When Toomre proposed his theory, there were no known long-lived indicators of mergers so that most ellipticals seemed 'clean', making it difficult to establish that all ellipticals originated through mergers.

Within the past ten years, however, improved instrumentation has revealed a wealth of detail in the cores and peripheries of ellipticals: photometrically and dynamically anomalous nuclei, rotating, for example, in the opposite sense to the whole galaxy; encircling shells of stars; disks and rings of neutral and ionized gas. These all seem to be the products of mergers, but for the most part of mergers between ellipticals and smaller companions, not necessarily between pairs of massive spiral galaxies. Models suggest that these features can persist ten times as long as it takes the original cores to fuse together.

In a previous extensive survey ${ }^{6}$, Schweizer and colleagues concluded that up to half of all bright ellipticals showed signs of a violent past (see figure). But even the others may have undergone mergers or may have been modified by acquiring material from other systems, only to have the post-interaction features erased by subsequent events. Schweizer et al. now claim' to have found a new signature of the addition of material for many of these ellipticals. The secret is in the stellar population of the galaxies. If the added matter consists of stars younger than those already present in the elliptical or of starforming gas, the post-merger galaxy will show spectral features of young stars. Such galaxies should be apparent on a plot of spectral character versus size.

In the past, the wide spread of spectral characteristics for ellipticals has obstructed the use of this approach. But by correlating these characteristics with a selection of post-merger structures (tidal tails and so on), Schweizer et al. have now devised a quantitative index $(\Sigma)$ which measures the amount of recent merging, they claim. When plotted against the difference from the mean luminosityspectral-line-strength relationship for ellipticals, large- $\Sigma$ systems do give the most discrepant points as expected for systems with a new injection of young stars.

As the young stars age, their influence on the global spectral properties of an elliptical will fade. Hence large deviations from the mean relationships should cor- relate with recent mergers introducing young stars - except that not all the postmerger features ( $\mathrm{X}$ and box structures, for example) examined by Schweizer et al. deteriorate with time; and for those that do change, the effect on $\Sigma$ is not always in the same direction. Also, not all mergers need deliver young stars or star-forming material. And because the outcome of a merger depends sensitively on initial conditions, mergers of a similar age could give different morphologies and hence different values of $\Sigma$.

So the correlations indicated by the $\Sigma$ index may be rather too simple for something so complex as galaxy merging. It will be worth taking a closer look at the sample examined by Schweizer et al. to see why they find such a good correlation between the $\Sigma$ index and stellar content. It may well be, for example, that ellipticals with spectacular structures are those resulting from collisions involving at least one spiral galaxy. As spirals have a good supply of young stars and star-forming materials, a correlation between $\Sigma$ and stellar content could follow.

On the modelling side, there are other difficulties with the idea that all ellipticals were formed by mergers of spiral galaxies. For one thing, several of the structural features seen in ellipticals, and used by Schweizer and colleagues, are actually suppressed in collisions between partners of equal mass, and are more readily produced in collisions between an existing massive elliptical and a smaller companion. So the observed features may indicate rather a shower of smaller systems on older ellipticals. And second, purely gravitional models of galaxy mergers do not explain why there seems to be a maximum size $\left(10^{12}\right.$ solar masses $)$ for galaxies. Nor do they explain the high density of stars in elliptical galaxies. Progress will depend on observations of high-redshift galaxies? (which we see as they were in earlier epochs of the Universe; the few imaged so far show chaotic structures indicative of a violent past) to elucidate the earliest mergers and more realistic models, incorporating hydrodynamic effects and the physics of star building.

Peter J. Quinn is at Mount Stromlo and Siding Spring Observatories, The Australian National University, Private Bag, Weston Creek PO, ACT 2611, Australia.

1. Schweizer, F. et al. Astrophys. J. 364, L33-L36 (1990) 2. Toomre, A. \& Toomre, J. Astrophys. J. 178, 623-666 (1972).

3. Van Albada, T.S. \& Van Gorkom, J.H. Astr. Astrophys. 54 121-126 (1977)

4. Toomre, A. in The Evolution of Galaxies and Stellar Populations (eds Tinsiey, B.M. \& Larson, R.B.) 401-426 (Yaie University Press, 1977)

5. Tremaine, S. in The Structure and Evolution of Normal Galaxies (eds Fall, S.M. \& Lynden-Bell, D.) 67-81 (Cambridge University Press, 1981).

6 Schweizer, F. \& Seitzer, P. Astrophys. J. 328, 88-92 (1988)

. Chambers, K.C., Miley, G.K. \& Van Breugel, W.J.M. Astro phys. J. 363, 21-39 (1990)

\section{Heavenly power}

LAST week Daedalus outlined his plan to levitate a ring of superconducting cable around the Earth. A permanent eastrunning current of a few million amps, repelled by the Earth's magnetic field, could hold the cable stably in space above the Equator. Radiation shields could keep the superconductor cold, while ionospheric drag kept it geosynchronous.

Applications would be legion. For a start, an equatorial 'space halo' would transform global communications. If positioned high enough, it could act as a passive but lossless multi-resonant loop-aerial, intercepting signals beamed up to it and reradiating them almost to the whole world. Fitted with active aerials and transponders as well, it could replace all expensive, short-lived telecommunication satellites.

Even at a mere 'auroral altitude' of 50-100 km, its Earth-horizon could be extended over most of the world by northand south-going side loops, which may be needed anyway for attitude stability. The halo could be stabilized still more firmly by connecting it to the ground with cable 'spokes'. Information, power or even equipment could then be sent up the cables. Telescopes or space-research instruments could be winched up and positioned around the halo by mobile 'halo runners'. Even a space-borne funicular railway might be possible. Lifted clear of the ground on a long cable, a funicular car could be swung across oceans and jungles by the halo runner high above, and lowered again at its destination.

A superconducting space-halo would be a powerful astronomical and geophysical instrument in its own right. As the largest possible iron-cored loop aerial, with no resistance and a lowest resonance at only a few hertz, it would open up a whole new region of high-sensitivity, low-frequency radioastronomy. Furthermore, at auroral altitude a cable carrying such a heavy steady current would powerfully perturb and attract the charged particles of the solar wind. It would be a permanent focus for auroral displays. All round the temperate zone, a glowing, shimmering, multicoloured arc would brighten the morning and evening sky, reducing street lighting bills and discouraging nocturnal crime. Better still, the space-halo would act as a lossless one-turn transformer for the a.c. components of the solar wind and the ionospheric electrojet currents. With suitable switching and controlling equipment, it could capture several gigawatts of natural, geophysical energy and transmit it down to Earth through its tethering cables. Daedalus is sending details to the US Department of Defense, which is already funding a visionary project to harness auroral currents.

David Jones 